\title{
Biomedical applications of polypyrrole microactuators: from single-cell clinic to microrobots
}

\author{
Edwin Jager, Charlotte Immerstrand, Karl-Eric Magnusson, Olle Inganäs and Ingemar \\ Lundström
}

\section{Linköping University Post Print}

\section{Tweet}

N.B.: When citing this work, cite the original article.

Original Publication:

Edwin Jager, Charlotte Immerstrand, Karl-Eric Magnusson, Olle Inganäs and Ingemar Lundström, Biomedical applications of polypyrrole microactuators: from single-cell clinic to microrobots, 2000, 1st Annual International, Conference On Microtechnologies in Medicine and Biology. 2000, 58-61.

http://dx.doi.org/10.1109/MMB.2000.893741

From the 1st Annual International IEEE-EMBS Special Topic Conference on Microtechnologies in Medicine \& Biology, Lyon, France, October 12-14, 2000

Copyright: IEEE

http://www.ieee.com/

Postprint available at: Linköping University Electronic Press

http://urn.kb.se/resolve?urn=urn:nbn:se:liu:diva-75794 


\title{
Biomedical Applications of Polypyrrole Microactuators: From Single-Cell Clinic to Microrobots
}

\author{
Edwin W.H. Jager (1,*), Charlotte Immerstrand (2), Karl-Erik Magnusson (2), \\ Olle Inganäs (1), Ingemar Lundström (1)
}

(1) Div. of Applied Physics, Dept. of Physics and Measurement Technology, Linköpings universitet, S-581 83 Linköping, Sweden, fax: +46-13-288969

(2) Div. of Medical Microbiology, Dept. of Health and Environment, Linköpings universitet, S-581 85 Linköping, Sweden, fax $+46-13-224789$

* Corresponding author, e-mail: edjag@ifm.liu.se

\begin{abstract}
Microtools that will be useful for the positioning and investigation of biological microstructures must operate under biologically relevant environments, such as cell culture media or blood plasma. They must also be comparatively strong, and preferably allow a muscle like mode of movement. This is given by a novel family of actuators based on conjugated polymers (like polypyrrole, PPy). By miniaturising these structures using standard photolithographic techniques, we can reduce the size down to 10-micrometer dimensions and build mechanically active microdevices. These can be moved and positioned by applying a potential to dope or undope the PPy. These novel structures are now being developed as a unique microactuator technology, suitable for operation in applications coupled to cell biology and biomedicine.
\end{abstract}

\section{INTRODUCTION}

The miniaturization of electronics has paved the way for miniaturization in other areas of technology, and has now reached the area of cell biology. Tools for investigations of single cells offer potential for new discoveries; tools for moving, positioning and mechanically manipulating cells and organelles and larger biological structures are necessary in the coming generations of microtechnology for medicine and biology. Microactuators of this kind must be able to operate in biological fluids, and must therefore be compatible with aqueous environments. Among the candidates for these functions we find laser tweezers, shape memory alloy structures and also polymeric electrochemical devices. Most of these are still operated with the help of the human eye, through some imaging method, but there is also a need for massive parallelism in the investigation of many individual elements, such as cell sorting. Here microtechnology may lend a hand, or rather a microrobot arm.

\section{PPY-MICROACTUATORS}

Conducting polymers like polypyrrole (PPy) are suitable materials for artificial muscles. PPy undergoes a volume change, driven by redox processes accompanied by ion movement. This volume change is reversible. Combining the PPy with a support and/or electrode layer into a bilayer we can make actuators [1-3]. These actuators are operated in a liquid electrolyte (salt solution).

Our microactuators are based on an Au/PPy bilayer in which the Au acts both as a structural layer and electrode. When we apply a positive potential to the Au electrode, we remove electrons from the PPy and oxidise it from its neutral state $\left(\mathrm{PPy}^{0}\right)$ to its oxidised state $\left(\mathrm{PPy}^{+}\right)$. This is a reversible process: we can reduce $\mathrm{PPy}$ from its oxidised state back to its neutral state by adding electrons. In analogy to the doping of silicon, the oxidised state is often called doped and the neutral state undoped. We use PPy doped with DBS $^{-}$(dodecylbenzenesulfonate) anions (PPy(DBS)) and operate the microactuators in an aqueous salt solution, usually NaDBS. However, the microactuators function also in other biological more relevant media, like blood plasma, urine, and cell culture media. During reduction of the PPy layer, small cations, in our case $\mathrm{Na}^{+}$ (surrounded by a layer of water molecules, the hydration shell), are inserted into the PPy to achieve charge neutrality in the PPy film. The film swells and thus the bilayer straightens. On oxidation, the $\mathrm{Na}^{+}$ions are expelled from the film, which shrinks and results in a bending motion [4]. We can summarise this redox reaction as follows:

$$
\mathrm{PPy}^{+}\left(\mathrm{DBS}^{-}\right)+\mathrm{e}^{-}+\mathrm{Na}^{+}(\mathrm{aq}) \leftrightarrow \mathrm{PPy}^{0}\left(\mathrm{DBS}^{-} \mathrm{Na}^{+}\right)
$$

For PPy(DBS) we achieve the neutral state at an applied voltage of $\mathrm{V}=-1 \mathrm{~V}$ vs. $\mathrm{Ag} / \mathrm{AgCl}$ and the oxidised state at $\mathrm{V}$ $=0 \mathrm{~V}$. By choosing a potential between the two limits (completely oxidised or completely reduced), we can position the microactuators at any intermediate position. It is also possible to move the actuators between intermediate positions, for instance from $-0.4 \mathrm{~V}$ to $-0.6 \mathrm{~V}$ and back to $0.4 \mathrm{~V}$.

\section{FABRICATION}

For the fabrication of the microactuators we used standard surface micromaching technology. As a substrate we used a Si wafer, and if we needed an insulating surface we used glass or a wafer with a $1 \mu \mathrm{m}$ thick thermal oxide layer. We could use two different fabrication methods to release the microstructures: differential adhesion [5] or a sacrificial layer.

The differential adhesion method is based on the poor adhesion of $\mathrm{Au}$ to $\mathrm{Si}$ or $\mathrm{SiO}_{2}$. On the substrate we thermally 
evaporated a thin layer (typically $200 \AA$ ) of $\mathrm{Cr}$ and Au: the adhesion layer. In this layer we patterned squares from which we etched the $\mathrm{Cr} / \mathrm{Au}$ until we had a bare Si surface again, see Fig. 1a. These squares would be the areas where the microactuators would be free. Next, we evaporated a second, thick (usually $1000 \AA$ ) Au layer: the structural layer (Fig. 1b.). Then we electropolymerised the PPy on the Au surface from a $0.1 \mathrm{M} \mathrm{NaDBS}$ and $0.1 \mathrm{M}$ pyrrole solution at a constant potential (between $0.5 \mathrm{~V}$ and $0.6 \mathrm{~V}$ vs. $\mathrm{Ag} / \mathrm{AgCl}$ ), as is shown in Fig. 1c. If the Au layer was 1000 $\AA$ thick, we grew a $1 \mu \mathrm{m}$ thick PPy layer. There were two ways to pattern the PPy. Either we grew the PPy on the whole $\mathrm{Au}$ covered wafer and then etched the PPy using reactive ion etching (RIE), or we covered the wafer with photoresist in which we had opened holes in which the PPy could grow. Next, we patterned photoresist and etched the structural Au to form the microactuators. As can be seen in Fig. 1c-d, a short piece of the microactuators laid on top of the $\mathrm{Cr} / \mathrm{Au}$ adhesion layer. This was the anchor that kept the actuator on the surface. Most of the actuator laid on the bare Si. After we stripped the photoresist used for the final Au etch, the actuators were finished, but still attached to the surface (Fig. 1d). They would release themselves during the first activation cycles. For more details on the fabrication of the PPy microactuators we refer to [6].

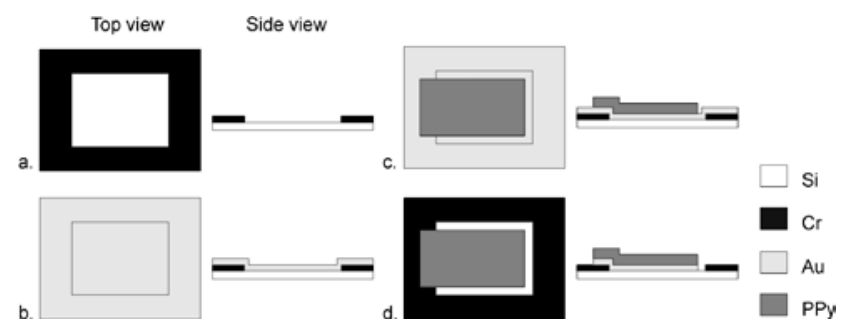

Fig. 1 The process steps to fabricate microactuators using the differential adhesion method. a. Depositing and patterning $\mathrm{Cr} / \mathrm{Au}$. b. Evaporating $\mathrm{Au}$

c. Electropolymerisation and patterning of PPy. d. Final result after etching the structural Au.

In some applications, like the microrobot, also other materials than Au were in contact with the Si substrate. As these materials adhered well to the $\mathrm{Si}$, we could not use the differential adhesion method. Therefore we also developed a sacrificial layer method. First, the sacrificial layer (for instance $\mathrm{SiO}_{2}$ on a $\mathrm{Si}$ substrate) was deposited and patterned, see

Fig. 2a. Then we thermally evaporated the Cr adhesion layer and the Au structural layer (Fig.2b.). Next, PPy was deposited in the same manner as above (Fig. 2c.). Then we patterned the microactuators by etching the Au and Cr. To release the structures, the sacrificial layer was removed. Fig. $2 \mathrm{~d}$ shows the final result. The actuators were now free and could be activated.

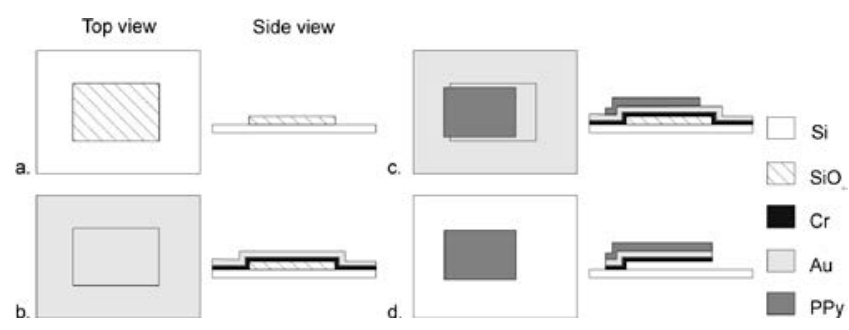

Fig. 2 Process steps for microactuator fabrication with the sacrificial layer method. a. Depositing and patterning the sacrificial layer, in this case CVD-SiO 2 b. Thermal evaporation of the $\mathrm{Cr}$ and $\mathrm{Au}$. c.

Electropolymerisation and patterning of PPy. d. Etching the $\mathrm{Au}$ and $\mathrm{Cr}$, and underetching the sacrificial layer to release the actuators.

We only described the fabrication of simple bilayer actuators (fingers), but it is more interesting to give the actuators some functionality and make devices. One can add rigid elements to make moving plates [7] and selfassembling boxes [8]. With other micromachined structures the possibilities increase. One could create valves [9], switches, grippers, robots, etc.

\section{MICROROBOT}

For the handling of small samples, like single cells, or microbeads coated with chemicals, proteins, etc., we have developed a microrobot arm [10]. The arm was built from $\mathrm{PPy} / \mathrm{Au}$ microactuator joints together with rigid elements (Fig. 3). Each joint could be separately addressed. By applying a voltage we controlled the bending angle of each joint and in this way we could control the movement of the microrobot.

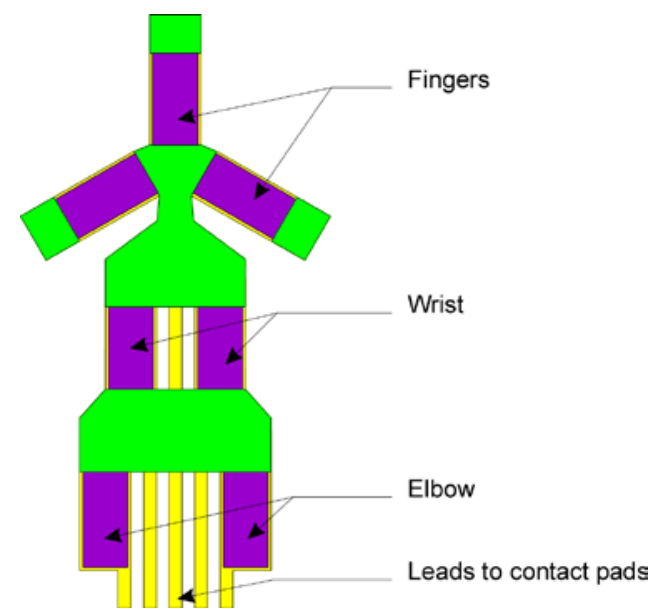

Fig 3. A top view picture of the microrobot. The robot has elbow joints, wrist, and 3 fingers.

We used the robot to grab, lift, and move $100 \mu \mathrm{m}$ glass beads. We have also made a track system of polyurethane at the base of the robot using microinjection moulding in capillaries (MIMIC). Using the robot arm, we then moved the bead sequentially all over the tracks, from track 4 , to 2 , to 1 , to 3 , and from the tracks to the base of the robot. Fig. 4 shows the robot with the bead on track 3 and 1 . The maximum displacement of the glass bead was $270 \mu \mathrm{m}$. 

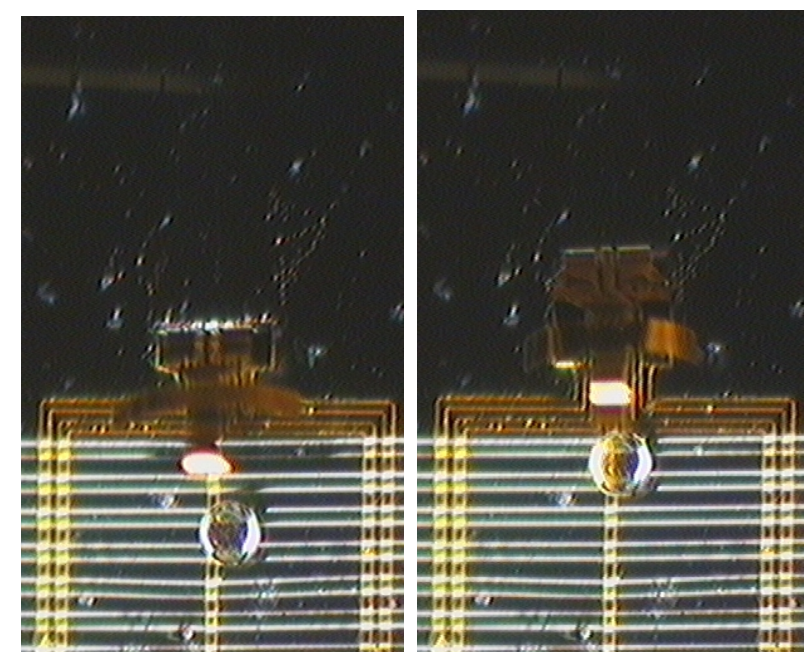

Fig.4 A microrobot arm (670 $\mu \mathrm{m}$ long and $240 \mu \mathrm{m}$ wide) moves a $100 \mu \mathrm{m}$ glass bead over a polyurethane track system made by MIMIC. Rather than glass beads single cells could be easily manipulated.

We believe that these microrobots are most interesting for operation in biological fluids. An application of the robot could be for multi station single cell diagnostics. The robot arm could arrest biological entities (single cells, bacteria, multi-cellular organisms, etc) from a sample and then transfer them sequentially to different measurement stations of a multisensor area as we demonstrated by transferring of the glass bead over the tracks. As an example an array of standing microrobots, where the fingers are treated with adhesion molecules, could be used to fish out given cells or bacteria in a sample, and then transfer them to the multisensor area. The testing could also be done by small additional structures on the microrobot itself, such as an extra electrode for electrical measurements or the electrodes could be located on the chip itself, e.g. in microvials (see section V), which could also enable single cell chemical modifications.

In medicine, the robot could be used as a tool for minimal invasive surgery. A small robot put on a catheter would increase the range of the surgeon.

\section{SINGLE-CELL CLINIC}

Not only do we want to manipulate single cells, we would also like to characterise them. For this purpose we are developing a (single-) cell clinic. This is a micrometersized cavity, or microvial, with a lid. The lid is activated by hinges made of PPy-microactuators, so that the cavity can be opened and closed on demand. Inside the cavity, sensors and actuators can be placed to stimulate the cells and record their responses (Fig. 5). In the cell clinics presented here we have put a pair of Au electrodes on the bottom of the microvial in order to do impedance measurements. In this way we can test cells in a confined volume.

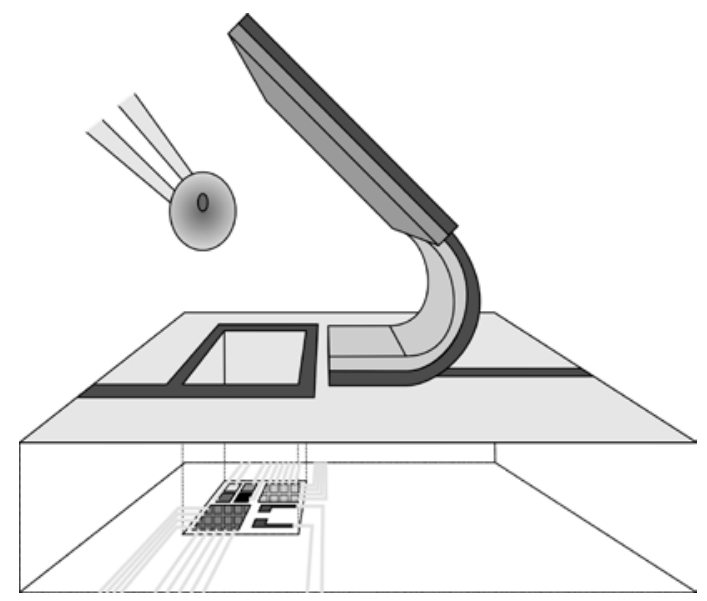

Fig. 5 A sketch of the sealable microvial including sensors and actuators on the bottom of the microvial. In the left corner a cell is introduced into the "cell clinic" in order to be studied

On a glass substrate we first patterned the Au electrode structure. Next, we made the microvials in a $20 \mu \mathrm{m}$ thick layer of SU-8. Then, we electropolymerised the PPy layers and finally we etched the complete layout, including the lids, wires and contact pads. Fig 6 (top) shows a photograph of four microvials of which one is closed by a lid. Fig 6 (bottom) shows microvials (without lids) loaded with fibroblasts.
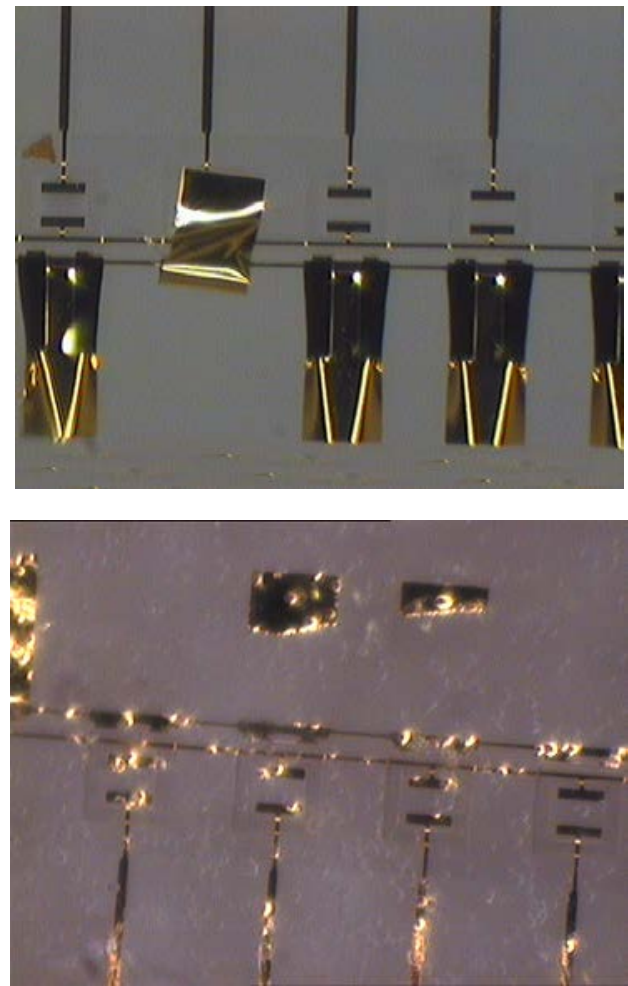

Fig.6 (top) A photograph of 4 microvials made of SU-8 (and therefore transparent) with 2 Au electrodes inside on a glass substrate. One microvial is closed with a lid. (bottom) Microvials loaded with fibroblasts (white dots). The second vial contains 3 cells. The microvials are $100 \mathrm{x}$ $100 \times 20 \mu \mathrm{m}^{3}$ ( $\mathrm{w} \times \mathrm{w} \times \mathrm{h}$ ), the lid is $150 \times 150 \mu \mathrm{m}^{2}$, and the electrodes are $20 \times 80 \mu \mathrm{m}^{2}$. 
As mentioned before, in our first generation of cell clinics we are using impedance measurements to characterise the cells. As a first characterisation of the devices we measured on $\mathrm{NaCl}$ solutions with physiologically relevant molarity. Fig. 7 shows the impedance measurements for 0.1-0.3 $\mathrm{M} \mathrm{NaCl}$ solution.

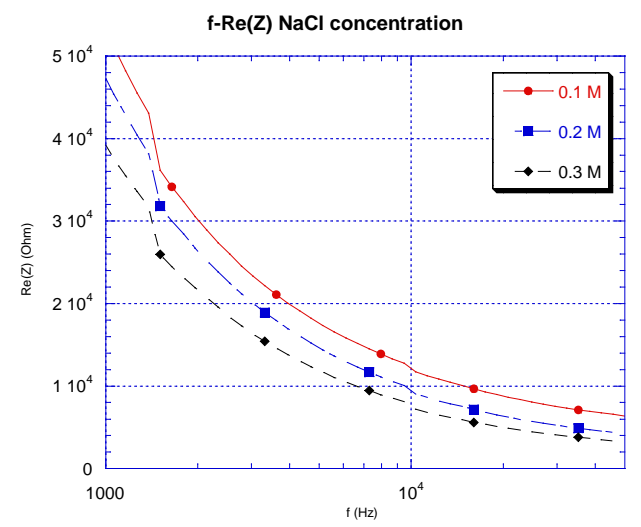

Fig 7 The real part of the impedance measured in a $\mathrm{NaCl}$ solution using the electrodes inside a (opened) microvial.

Next we loaded the microvials with fibroblasts and measured the impedance. First we measured the impedance on the sucrose medium to get a base line, then added the fibroblasts, and finally lysed the cells by adding triton X100. After cleaning the device, we repeated the measurement series with the same solutions, however instead of adding cells, we added a dummy solution, which was similar to the cell solution except that it did not contain any cells. As can be seen in fig. 8 there is a difference between the impedance of the dummy and cell suspension. This difference we attribute to the presence of the fibroblasts.

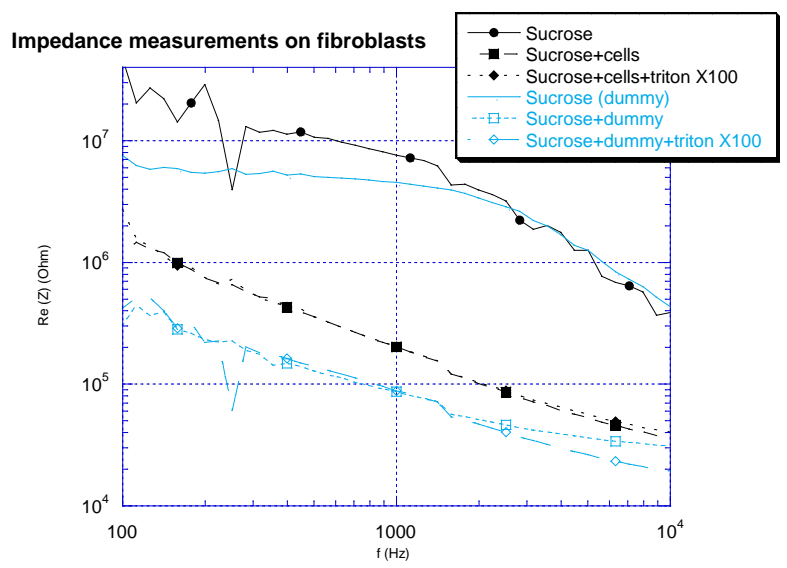

Fig 8.The real part of the impedance measured on fibroblasts using the electrodes in (opened) microvials

\section{ACKOWLEDGEMENT}

E.W.H.J. and C.I. are PhD-students enrolled in the graduate school Forum Scientum and wish to thank the Swedish Foundation for Strategic Research (SSF) for its financial support.

\section{REFERENCES}

1 R. H. Baughman, Conducting polymer artificial muscles, Synth. Met., 78 (1996) 339-353

2 T. F. Otero and J. M. Sansinena, Artificial muscles based on conducting polymers, Bioelectrochemistry and Bioenergetics, 38 (1995) 411-414

3 A. Della Santa, D. De Rossi, and A. Mazzoldi, Characterization and modelling of a conducting polymer muscle-like linear actuator, Smart Mater. Struct., 6 (1997) 23-24

4 Q. Pei and O. Inganäs, Electrochemical applications of the bending beam method. 2. Electroshrinking and slow relaxation in polypyrrole, J. Phys. Chem., 97 (1993) 6034-6041

5 E. Smela, O. Inganäs, and I. Lundström, Differential adhesion method for microstructure release: an alternative to the sacrificial layer, Transducers '95, Stockholm, Sweden, 1995, pp. 218-219

6 E. Smela, Microfabrication of PPy microactuators and other conjugated polymer devices, J. Micromech. Microeng., 9 (1999) 1-18

7 E. Smela, M. Kallenbach, and J. Holdenried, Electrochemically driven polypyrrole bilayers for moving and positioning bulk micromachined silicon plates, J. Microelectromech. Syst., 8 (1999) 373-383

8 E. Smela, O. Inganäs, and I. Lundström, Self-opening and closing boxes and other micromachined folding structures, Transducers '95, Stockholm, Sweden, 1995, pp. 350-351

9 F. Pettersson, E. W. H. Jager, and O. Inganäs, Surface Micromachined Polymer Actuators as Valves in PDMS Microfluidic System, IEEE-EMBS Special Topic Conference on Microtechnologies in Medicine \& Biology, Lyon, France, October 12-14, 2000, 2000,

10 E. W. H. Jager, O. Inganäs, and I. Lundström, Microrobots for Micrometer-Size Objects in Aqueous Media: Potential Tools for Single Cell Manipulation, Science, 288 (2000) 2335-2338 